\title{
Consecutive Quadratic Residues and Primitive Roots in the Sequences Formed by Twice-differentiable Functions
}

\author{
Mengyao Jing and Huaning Liu*
}

Abstract. In this paper we bound character sums of the shape

$$
\sum_{n \leq N} \chi_{1}(\lfloor f(n)\rfloor) \chi_{2}(\lfloor f(n+l)\rfloor)
$$

where $\chi_{1}$ and $\chi_{2}$ are non-principal multiplicative characters modulo a prime $p, f(x)$ is a real-valued, twice-differentiable function satisfying a certain condition on $f^{\prime \prime}(x)$, and $l$ is a positive integer. As an immediate application, we obtain some distribution properties of consecutive quadratic residues and consecutive primitive roots in Piatetski-Shapiro sequences $\left\lfloor n^{c}\right\rfloor$ with $c \in(1,4 / 3)$.

\section{Introduction}

The Piatetski-Shapiro sequence associated with $c \in(1,2)$ is defined by $\left(\left\lfloor n^{c}\right\rfloor\right)_{n \in \mathbb{N}}$, where $\lfloor x\rfloor$ denotes the floor function. Investigations into arithmetic properties of these types of sequences have attracted wide interest. For example, many papers have been written on the least quadratic non-residues in Piatetski-Shapiro sequences (see $[1,2,9,12]$ ).

Later, Banks and Shparlinski [5] focused on more general sequences. Let $\kappa$ be a real number with $2 / 3<\kappa<1$ and let $f(x)$ be a real-valued, twice-differentiable function such that

$$
\lim _{x \rightarrow \infty} \frac{\log f^{\prime \prime}(x)}{\log x}=-\kappa .
$$

The constant $\kappa$ corresponds to $2-c$ in the case of the Piatetski-Shapiro sequence $\left(\left\lfloor n^{c}\right\rfloor\right)_{n \in \mathbb{N}}$. They studied character sums over the integer sequences $(\lfloor f(n)\rfloor)_{n \in \mathbb{N}}$ and obtained the following result.

Received October 14, 2021; Accepted December 15, 2021.

Communicated by Liang-Chung Hsia.

2020 Mathematics Subject Classification. 11L40, 11A07, 11B83.

Key words and phrases. quadratic residue, primitive root, twice-differentiable function, character sum. This work is supported by National Natural Science Foundation of China under Grant No. 12071368, and the Science and Technology Program of Shaanxi Province of China under Grant Nos. 2019JM-573 and 2020JM-026.

*Corresponding author. 
Proposition 1.1. Let $p$ be an odd prime, $\kappa$ and $\varepsilon$ be fixed real numbers such that

$$
\frac{2}{3}<\kappa<1 \quad \text { and } \quad 0<\varepsilon<\frac{3 \kappa-2}{2 \kappa(2-\kappa)}
$$

and let $f(x)$ be a real-valued, twice-differentiable function satisfying (1.1). Then, for all non-principal characters $\chi$ modulo $p$ and all integers $N$ with

$$
p^{1 /(2 \kappa)+\varepsilon} \leq N \leq p^{1 /(2-\kappa)}
$$

the uniform bound

$$
\sum_{n \leq N} \chi(\lfloor f(n)\rfloor) \ll_{\varepsilon, f} N p^{-\delta}
$$

holds with some constant $\delta>0$ that depends at most on $\varepsilon$ and $f$.

Following their proof, we can prove that one may take $\delta=2^{-9} \varepsilon^{2} \kappa^{4}$ in the above. From Proposition 1.1 we derive that for every sufficiently larger prime $p$, there is a positive integer $n \leq p^{1 /(2 \kappa)+o(1)}$ such that $\lfloor f(n)\rfloor$ is a quadratic non-residue modulo $p$. Furthermore, for $1<c<4 / 3$, there is a positive integer $n \leq p^{1 /(4-2 c)+o(1)}$ such that $\left\lfloor n^{c}\right\rfloor$ is a quadratic non-residue modulo $p$.

On the other hand, many scholars studied the distribution of consecutive quadratic residues and primitive roots modulo $p$ (see $[6,8,10,11,15,16]$ ). For instance, Tanti and Thangadurai [15] proved that for all $p \geq 7$ (respectively for $p \geq 5$ ), there is an integer $m$ such that $m$ and $m+1$ are quadratic residues (respectively for quadratic non-residues) modulo $p$. We remark that this is loosely related to Artin's primitive root conjecture in the sense that one certainly has a pair $m, m+1$ of consecutive quadratic non-residues modulo $p$ if 2 is a primitive root modulo $p$ and this is believed to happen infinitely often.

In this paper we study the distribution of consecutive quadratic residues and primitive roots in the sequences $(\lfloor f(n)\rfloor)_{n \in \mathbb{N}}$, where $f(x)$ is a real-valued, twice-differentiable function. The crucial tool is to bound character sums of the shape

$$
S_{f}\left(\chi_{1}, \chi_{2} ; N, l\right)=\sum_{n \leq N} \chi_{1}(\lfloor f(n)\rfloor) \chi_{2}(\lfloor f(n+l)\rfloor),
$$

where $l$ is a positive integer. We shall prove that the bound in Proposition 1.1 is also applicable to 1.2 if $l$ is small enough. Our results are as follows and the proof method due to 5,14 . For convenience, we denote

$$
\theta=2^{-9} \varepsilon^{2} \kappa^{4}
$$

throughout this paper. 
Theorem 1.2. Let $\kappa$ and $\varepsilon$ be fixed real numbers such that

$$
\frac{2}{3}<\kappa<1 \quad \text { and } \quad 0<\varepsilon<\frac{3 \kappa-2}{2 \kappa(2-\kappa)},
$$

and let $f(x)$ be a real-valued, twice-differentiable function such that

$$
\lim _{x \rightarrow \infty} \frac{\log f^{\prime \prime}(x)}{\log x}=-\kappa .
$$

Then, for all non-principal multiplicative characters $\chi_{1}, \chi_{2}$ modulo $p$ and all integers $l$, $N$ with

$$
p^{1 /(2 \kappa)+\varepsilon} \leq N \leq p^{1 /(2-\kappa)} \quad \text { and } \quad 0<l \leq p^{\varepsilon / 2},
$$

the uniform bound

$$
S_{f}\left(\chi_{1}, \chi_{2} ; N, l\right) \ll_{\varepsilon, f} N p^{-\delta}
$$

holds with some constant $\delta>0$ that depends at most on $\varepsilon$ and $f$. In particular, one may take $\delta=\theta$.

Theorem 1.3. Suppose that $\varepsilon, \kappa, f(x), l$ and $N$ are defined as in Theorem 1.2. Let $Q_{f}(N, l)$ denote the number of positive integers $n \leq N$ such that $\lfloor f(n)\rfloor$ and $\lfloor f(n+l)\rfloor$ are quadratic residues modulo $p$. Then we have

$$
Q_{f}(N, l)=\frac{N}{4}+O_{\varepsilon, f}\left(N p^{-\theta}\right)
$$

where $\theta$ is defined as in (1.3).

Theorem 1.4. Suppose that $\varepsilon, \kappa, f(x), l$ and $N$ are defined as in Theorem 1.2. Let $G_{f}(N, l)$ denote the number of positive integers $n \leq N$ such that $\lfloor f(n)\rfloor$ and $\lfloor f(n+l)\rfloor$ are primitive roots modulo $p$. Then we have

$$
G_{f}(N, l)=\left(\frac{\phi(p-1)}{p-1}\right)^{2} N+O_{\varepsilon, f}\left(\left(\frac{\phi(p-1)}{p-1}\right)^{2} 4^{\omega(p-1)} N p^{-\theta}\right),
$$

where $\theta$ is defined as in (1.3), $\phi$ is the Euler function and $\omega(q)$ denotes the number of distinct prime factors of $q$.

As an immediate application of Theorems 1.3 and 1.4, we obtain bounds on the least consecutive quadratic residues and primitive roots in Piatetski-Shapiro sequences.

Corollary 1.5. For every sufficiently large prime $p$ and for $1<c<4 / 3$, there is a positive integer $n \leq p^{1 /(4-2 c)+o(1)}$ such that $\left\lfloor n^{c}\right\rfloor$ and $\left\lfloor(n+1)^{c}\right\rfloor$ are quadratic residues modulo $p$.

Corollary 1.6. For every sufficiently large prime $p$ and for $1<c<4 / 3$, there is a positive integer $n \leq p^{1 /(4-2 c)+o(1)}$ such that $\left\lfloor n^{c}\right\rfloor$ and $\left\lfloor(n+1)^{c}\right\rfloor$ are primitive roots modulo $p$.

The rest of this paper is structured as follows. First, in Section 2 we recall some properties of discrepancy and estimate certain double character sums. In Section 3 we bound the sum (1.2) over short intervals and this bound will be applied to prove Theorem 1.2 in Section 4. Finally, in Section 5 we prove Theorems 1.3 and 1.4 . 


\section{Preliminaries}

The first purpose of this section is to introduce some information about discrepancy. The discrepancy $D(N)$ of a sequence of (not necessarily distinct) real numbers $x_{1}, \ldots, x_{N} \in$ $[0,1)$ is defined by

$$
D(N)=\sup _{\mathcal{I} \subseteq[0,1)}\left|\frac{V(\mathcal{I}, N)}{N}-\right| \mathcal{I}||,
$$

where the supremum is taken over all intervals $\mathcal{I}=[a, b) \subseteq[0,1), V(\mathcal{I}, N)$ is the number of integers $n \leq N$ such that $x_{n} \in \mathcal{I}$ and $|\mathcal{I}|$ is the length of $\mathcal{I}$.

Banks and Shparlinski studied the discrepancy of fractional parts of twice-differentiable functions by using the Erdős-Turán inequality. The following result is taken from 5 , Lemma 3.2].

Lemma 2.1. Assume that $f(x)$ is a real-valued, twice-differentiable function such that

$$
0<\alpha \leq f^{\prime \prime}(x) \leq \alpha \beta \quad\left(\text { or } \quad 0<\alpha \leq-f^{\prime \prime}(x) \leq \alpha \beta\right)
$$

throughout the the interval $[K, K+L]$, where $L \geq 1$. Then the discrepancy $D_{f}(K, K+L)$ of the sequence of fractional parts $(\{f(n)\})_{n \in(K, K+L] \cap \mathbb{Z} \text { satisfies }}$

$$
D_{f}(K, K+L) \ll \alpha^{1 / 3} \beta^{2 / 3}+L^{-1} \alpha^{-1 / 2} .
$$

The second purpose of this section is to bound certain double character sums in Lemmas 2.3 and 2.4. In order to prove our results, we present Weil's bound [13, Theorem $2 \mathrm{C}$ ] on character sums in Lemma 2.2,

Lemma 2.2. Let $\mathbb{F}_{q}$ be a finite field and let $\chi$ be a multiplicative character of order $w>1$ of $\mathbb{F}_{q}$. Let $F(x) \in \mathbb{F}_{q}[x]$ be a non-constant polynomial which is not a constant times of a $w$-th power and let $s$ denote the number of distinct zeros of $F(x)$ in $\overline{\mathbb{F}}_{q}$. Then we have

$$
\left|\sum_{x \in \mathbb{F}_{q}} \chi(F(x))\right| \leq(s-1) \sqrt{q} .
$$

Lemma 2.3. Let $p$ be an odd prime, $t$ be an integer with $1 \leq t \leq p-1$ and let $\chi_{1}, \chi_{2}$ be non-principal characters modulo $p$. Suppose that $\mathcal{U}, \mathcal{V}$ are subsets of $\mathbb{F}_{p}$ of cardinalities $\# \mathcal{U}=U$ and $\# \mathcal{V}=V$. Then, for an arbitrary fixed integer $k$, for any complex numbers $a_{u}, b_{v}(u \in \mathcal{U}, v \in \mathcal{V})$ we have

$$
\sum_{u \in \mathcal{U}} \sum_{v \in \mathcal{V}} a_{u} b_{v} \chi_{1}(u+v) \chi_{2}(u+v+t) \ll_{k} A B U^{1-1 /(2 k)}\left(V^{1 / 2} p^{1 /(2 k)}+V p^{1 /(4 k)}\right),
$$

where

$$
A=\max _{u \in \mathcal{U}}\left|a_{u}\right| \quad \text { and } \quad B=\max _{v \in \mathcal{V}}\left|b_{v}\right|
$$


Proof. Denote the left side of 2.2 by $\Gamma$. Using the Hölder inequality we have

$$
\begin{aligned}
|\Gamma|^{2 k} & \leq A^{2 k} U^{2 k-1} \sum_{u \in \mathcal{U}}\left|\sum_{v \in \mathcal{V}} b_{v} \chi_{1}(u+v) \chi_{2}(u+v+t)\right|^{2 k} \\
& \leq A^{2 k} U^{2 k-1} \sum_{u \in \mathbb{F}_{p}}\left|\sum_{v \in \mathcal{V}} b_{v} \chi_{1}(u+v) \chi_{2}(u+v+t)\right|^{2 k} .
\end{aligned}
$$

Let $\chi_{*}$ be a character modulo $p$ of order $p-1$. Then, every character $\chi$ modulo $p$ can be expressed as $\chi=\chi_{*}^{\delta_{0}}$, where $\delta_{0}$ is an integer with $1 \leq \delta_{0} \leq p-1$. Note that $\chi_{1}$ and $\chi_{2}$ are non-principal characters, then there exist some $1 \leq \delta_{1}, \delta_{2} \leq p-2$ such that

$$
\chi_{1}=\chi_{*}^{\delta_{1}} \quad \text { and } \quad \chi_{2}=\chi_{*}^{\delta_{2}}
$$

It follows that

$$
\begin{aligned}
|\Gamma|^{2 k} \leq & A^{2 k} U^{2 k-1} \sum_{u \in \mathbb{F}_{p}}\left|\sum_{v \in \mathcal{V}} b_{v} \chi_{*}\left((u+v)^{\delta_{1}}(u+v+t)^{\delta_{2}}\right)\right|^{2 k} \\
= & A^{2 k} U^{2 k-1} \sum_{v_{1}, \ldots, v_{k} \in \mathcal{V}} \sum_{v_{k+1}, \ldots, v_{2 k} \in \mathcal{V}} b_{v_{1}} \cdots b_{v_{k}} \overline{b_{v_{k+1}}} \cdots \overline{b_{v_{2 k}}} \\
& \times \sum_{u \in \mathbb{F}_{p}} \chi_{*}\left(\prod_{i=1}^{k}\left(u+v_{i}\right)^{\delta_{1}}\left(u+v_{i}+t\right)^{\delta_{2}}\right) \overline{\chi_{*}}\left(\prod_{j=k+1}^{2 k}\left(u+v_{j}\right)^{\delta_{1}}\left(u+v_{j}+t\right)^{\delta_{2}}\right) .
\end{aligned}
$$

For $(m, p)=1$, let $\bar{m}$ denote the multiplicative inverse of $m$ modulo $p$. If $\left(\left(u+v_{j}\right)(u+\right.$ $\left.\left.v_{j}+t\right), p\right)=1$ we have

$$
\begin{aligned}
\overline{\chi_{*}}\left(\left(u+v_{j}\right)^{\delta_{1}}\left(u+v_{j}+t\right)^{\delta_{2}}\right) & =\chi_{*}\left(\overline{\left(u+v_{j}\right)^{\delta_{1}}\left(u+v_{j}+t\right)^{\delta_{2}}}\left(u+v_{j}\right)^{p-1}\left(u+v_{j}+t\right)^{p-1}\right) \\
& =\chi_{*}\left(\left(u+v_{j}\right)^{p-1-\delta_{1}}\left(u+v_{j}+t\right)^{p-1-\delta_{2}}\right) .
\end{aligned}
$$

Thus we have

$$
|\Gamma|^{2 k} \ll A^{2 k} B^{2 k} U^{2 k-1} \sum_{v_{1}, \ldots, v_{2 k} \in \mathcal{V}}\left|\sum_{u \in \mathbb{F}_{p}} \chi_{*}(H(u))\right|,
$$

where

$$
H(u)=\prod_{i=1}^{k}\left(u+v_{i}\right)^{\delta_{1}}\left(u+v_{i}+t\right)^{\delta_{2}} \prod_{j=k+1}^{2 k}\left(u+v_{j}\right)^{p-1-\delta_{1}}\left(u+v_{j}+t\right)^{p-1-\delta_{2}} .
$$

Define the subset $\Omega \subseteq \mathcal{V}^{2 k}$ as

$\Omega=\left\{\left(v_{1}, \ldots, v_{2 k}\right) \in \mathcal{V}^{2 k}: H(u)\right.$ is the $(p-1)$-th power of a polynomial over $\left.\mathbb{F}_{p}\right\}$ 
By Lemma 2.2, the inner sum in 2.3 can be estimated as

$$
\sum_{u \in \mathbb{F}_{p}} \chi_{*}(H(u))= \begin{cases}O(p) & \text { if }\left(v_{1}, \ldots, v_{2 k}\right) \in \Omega \\ O_{k}(\sqrt{p}) & \text { if }\left(v_{1}, \ldots, v_{2 k}\right) \in \mathcal{V}^{2 k} \backslash \Omega .\end{cases}
$$

Therefore, from $(2.3$ and 2.4 we immediately get

$$
|\Gamma|^{2 k} \ll_{k} A^{2 k} B^{2 k} U^{2 k-1}\left((\# \Omega) p+V^{2 k} \sqrt{p}\right) .
$$

It remains to treat the cardinality of $\Omega$. We construct a new polynomial $G(u)$ as

$$
G(u)=\prod_{i=1}^{2 k}\left(u+v_{i}\right)\left(u+v_{i}+t\right)
$$

and define the subset $\Omega_{1} \subseteq \mathcal{V}^{2 k}$ as

$$
\Omega_{1}=\left\{\left(v_{1}, \ldots, v_{2 k}\right) \in \mathcal{V}^{2 k}: G(u) \text { has no simple root }\right\}
$$

It is clear that $G(u)$ and $H(u)$ have the same roots. Since $1 \leq \delta_{1}, \delta_{2} \leq p-2$, then $G(u)$ having a simple root implies that $H(u)$ can not be the $(p-1)$-th power of a polynomial over $\mathbb{F}_{p}$. It follows that $\Omega \subseteq \Omega_{1}$. Next, we shall prove that the cardinality of $\Omega_{1}$ satisfies

$$
\# \Omega \leq \# \Omega_{1} \leq(k+1) k^{2}(2 k)^{2 k} V^{k}
$$

For every $\left(v_{1}, \ldots, v_{2 k}\right) \in \Omega_{1}$, then for $v \in\left\{v_{1}, \ldots, v_{2 k}\right\}$ we write

$$
\eta(v)=\#\left\{1 \leq i \leq 2 k: v_{i}=v\right\}
$$

Thus, we can divide $\left\{v_{1}, \ldots, v_{2 k}\right\}$ into two disjoint sets:

$$
\left\{v_{1}, \ldots, v_{2 k}\right\}=\mathcal{C} \cup \mathcal{D}
$$

where

$$
\mathcal{C}=\left\{c \in\left\{v_{1}, \ldots, v_{2 k}\right\}: \eta(c) \geq 2\right\}, \quad \mathcal{D}=\left\{d \in\left\{v_{1}, \ldots, v_{2 k}\right\}: \eta(d)=1\right\}
$$

We denote the cardinality of $\mathcal{D}$ by $D$ and write $\mathcal{D}=\left\{d_{1}, \ldots, d_{D}\right\}$. Consequently, the cardinality of $\mathcal{C}$ satisfies

$$
\# \mathcal{C} \leq\lfloor(2 k-D) / 2\rfloor
$$

Therefore,

$$
G(u)=\left(\prod_{c \in \mathcal{C}}(u+c)^{\eta(c)}(u+c+t)^{\eta(c)}\right)\left(\prod_{d \in \mathcal{D}}(u+d)(u+d+t)\right) .
$$


Since $G(u)$ has no simple root, for every $d_{i} \in \mathcal{D}$ there exists some $c \in \mathcal{C}$ or $d_{j} \in \mathcal{D}$ such that

$$
d_{i} \equiv d_{j}+t \quad(\bmod p) \quad \text { or } \quad d_{i} \equiv c+t \quad(\bmod p) .
$$

Hence, for fixed $\mathcal{C}$, we only need to choose $\lfloor D / 2\rfloor$ values from $\mathcal{V}$ at most and we write these values to form a set $\mathcal{J}$. Then, every $d \in \mathcal{D}$ takes a value from $\mathcal{J}, \mathcal{J}+t$ and $\mathcal{C}+t$. Thus we have

$$
\begin{aligned}
\# \Omega_{1} & \leq \sum_{D=0}^{2 k} \sum_{i=1}^{\lfloor(2 k-D) / 2\rfloor}\left(\begin{array}{l}
V \\
i
\end{array}\right) i^{2 k-D} \sum_{j=1}^{\lfloor D / 2\rfloor}\left(\begin{array}{l}
V \\
j
\end{array}\right)\left(2 j+\frac{2 k-D}{2}\right)^{D} \\
& \leq \sum_{D=0}^{2 k} \sum_{i=1}^{\lfloor(2 k-D) / 2\rfloor}\left(\begin{array}{l}
V \\
i
\end{array}\right) i^{2 k-D} \frac{D}{2}\left(k+\frac{D}{2}\right)^{D} V^{D / 2} \\
& \leq \sum_{D=0}^{2 k} \frac{D}{2}\left(k-\frac{D}{2}\right)\left(k-\frac{D}{2}\right)^{2 k-D}\left(k+\frac{D}{2}\right)^{D} V^{k} \\
& \leq(k+1) k^{2}(2 k)^{2 k} V^{k} .
\end{aligned}
$$

This proves (2.6). Now combining $(2.5)$ and $(2.6)$ we immediately get Lemma 2.3 .

Lemma 2.4. Assume the hypotheses of Lemma 2.3. Then, for any $\varepsilon>0, U \geq p^{1 / 2+\varepsilon}$ and any $V \geq p^{\varepsilon}$, we have

$$
\sum_{u \in \mathcal{U}} \sum_{v \in \mathcal{V}} a_{u} b_{v} \chi_{1}(u+v) \chi_{2}(u+v+t) \ll_{\varepsilon} A B U V p^{-\varepsilon^{2} /(1+2 \varepsilon)} .
$$

Proof. Taking $k=\lceil 1 /(2 \varepsilon)\rceil$ we get $V \geq p^{1 /(2 k)}$. Then, the right side of 2.2$)$ satisfies

$$
A B U^{1-1 /(2 k)}\left(V^{1 / 2} p^{1 /(2 k)}+V p^{1 /(4 k)}\right) \leq A B U^{1-1 /(2 k)} V p^{1 /(4 k)} \leq A B U V p^{-\varepsilon /(2 k)} .
$$

Since $U \leq p$, then we have $\varepsilon \leq 1 / 2$. Therefore, $\varepsilon /(2 k) \geq \varepsilon^{2} /(1+2 \varepsilon)$. This proves Lemma 2.4.

\section{Character sums over short intervals}

Throughout this section we suppose that $p$ is a sufficiently large prime. Like the technical method in 5], our bound for $S_{f}\left(\chi_{1}, \chi_{2} ; N, l\right)$ is related to bounds for more general sums

$$
S_{f}\left(\chi_{1}, \chi_{2} ; K, L, l\right)=\sum_{K<n \leq K+L} \chi_{1}(\lfloor f(n)\rfloor) \chi_{2}(\lfloor f(n+l)\rfloor),
$$

where $K$ and $L$ are sufficiently large real numbers that satisfy certain conditions. Our result is as follows. 
Theorem 3.1. Fix $\varepsilon>0$ and $2 / 3<\kappa<1$. Let $f(x)$ be a real-valued, twice-differentiable function such that

$$
\lim _{x \rightarrow \infty} \frac{\log f^{\prime \prime}(x)}{\log x}=-\kappa
$$

Then, for all non-principal characters $\chi_{1}, \chi_{2}$ modulo $p$, all positive integers $l$ with $l \leq p^{\varepsilon / 2}$ and all real numbers $K, L$ that satisfy the inequalities

$$
\text { (i) } K^{\kappa-\varepsilon} \geq L \geq K^{\kappa / 2} p^{\varepsilon}, \quad \text { (ii) } K \leq p^{1 /(2-\kappa)}, \quad \text { (iii) } L \geq p^{1 / 2+\varepsilon} \text {, }
$$

we have

$$
S_{f}\left(\chi_{1}, \chi_{2} ; K, L, l\right) \ll_{\varepsilon, f} L p^{-\varepsilon^{2} / 32} .
$$

Proof. Put $H=\left\lceil 2 p^{\varepsilon / 2}\right\rceil$. The assumptions of Theorem 3.1 imply that

$$
0<\alpha \leq f^{\prime \prime}(x) \leq \alpha \beta, \quad K \leq x \leq K+L+H+l
$$

with real numbers $\alpha$ and $\beta$ of the size

$$
\alpha=K^{-\kappa+o_{f}(1)} \quad \text { and } \quad \beta=K^{o_{f}(1)},
$$

where the implied constants depend on $f$. For any integers $n \in(K, K+L]$ and $h \in[0, H-1]$ we have

$$
f(n+h)-f(n)-h f^{\prime}(n)=\int_{n}^{n+h} f^{\prime \prime}(u)(n+h-u) d u
$$

Thus we have

$$
0 \leq f(n+h)-f(n)-h f^{\prime}(n) \leq 0.5 H^{2} \alpha \beta
$$

Note that

$$
0 \leq f^{\prime}(n)-f^{\prime}(K)=\int_{K}^{n} f^{\prime \prime}(u) d u \leq L \alpha \beta
$$

Therefore,

$$
0 \leq f(n+h)-f(n)-h f^{\prime}(K) \leq 2 H L \alpha \beta
$$

Similarly we obtain

$$
0 \leq f(n+h+l)-f(n)-(h+l) f^{\prime}(K) \leq 2(H+l) L \alpha \beta
$$

Let $\Delta=p^{-\varepsilon / 4}$ with $0<\Delta<1 / 2$, and let $D=\left\lceil\Delta^{-1}\right\rceil$. By the pigeonhole principle there is an integer $j_{0} \in\{0, \ldots, D-1\}$ such that the fractional part $\left\{h f^{\prime}(K)\right\}$ lies in the interval $\left[j_{0} / D,\left(j_{0}+1\right) / D\right)$ for at least $H / D$ values of $h \in\{0,1, \ldots, H-1\}$. Let $\mathcal{H}$ be the set of such integers $h$ and let $\xi=j_{0} / D$. Then we have

$$
\mathcal{H}=\left\{h \in[0, H-1] \cap \mathbb{Z}:\left\{h f^{\prime}(K)-\xi\right\}<1 / D\right\},
$$


$0.5 \Delta<1 / D \leq \Delta$ and the cardinality of $\# \mathcal{H}$ satisfies

$$
\# \mathcal{H} \geq H / D \geq 0.5 \Delta H \geq p^{\varepsilon / 4}
$$

Next, we define two subsets $\mathcal{N}_{1}, \mathcal{N}_{2} \subseteq(K, K+L]$ as

$$
\begin{aligned}
& \mathcal{N}_{1}=\{n \in(K, K+L] \cap \mathbb{N}:\{f(n)+\xi\}<1-\Delta-2(H+l) L \alpha \beta\}, \\
& \mathcal{N}_{2}=\left\{n \in(K, K+L] \cap \mathbb{N}:\left\{f(n)+l f^{\prime}(K)+\xi\right\}<1-\Delta-2(H+l) L \alpha \beta\right\} .
\end{aligned}
$$

We also define their complementary sets as

$$
\mathcal{N}_{1}^{c}=((K, K+L] \cap \mathbb{N}) \backslash \mathcal{N}_{1} \quad \text { and } \quad \mathcal{N}_{2}^{c}=((K, K+L] \cap \mathbb{N}) \backslash \mathcal{N}_{2}
$$

Let $\mathcal{N}=\mathcal{N}_{1} \cap \mathcal{N}_{2}$. Hence, 3.1 and 3.2 imply that

$$
\lfloor f(n+h)\rfloor=\lfloor f(n)+\xi\rfloor+\left\lfloor h f^{\prime}(K)-\xi\right\rfloor
$$

and

$$
\lfloor f(n+h+l)\rfloor=\left\lfloor f(n)+l f^{\prime}(K)+\xi\right\rfloor+\left\lfloor h f^{\prime}(K)-\xi\right\rfloor
$$

hold for every pair $(n, h) \in \mathcal{N} \times \mathcal{H}$.

Now we estimate the cardinality of $\mathcal{N}^{c}=((K, K+L] \cap \mathbb{N}) \backslash \mathcal{N}$. It is clear that

$$
\mathcal{N}^{c}=\left(\mathcal{N}_{1} \cap \mathcal{N}_{2}\right)^{c}=\mathcal{N}_{1}^{c} \cup \mathcal{N}_{2}^{c}
$$

Thus we have

$$
\# \mathcal{N}^{c} \leq \# \mathcal{N}_{1}^{c}+\# \mathcal{N}_{2}^{c}
$$

Write $f_{1}(x)=f(x)+\xi$ and $f_{2}(x)=f(x)+l f^{\prime}(K)+\xi$. Recall that $D_{f}(K, K+L)$ is

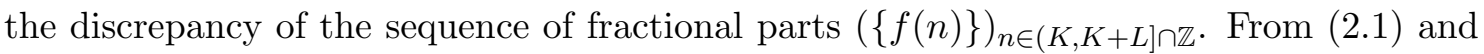
Lemma 2.1 we have

$$
\begin{aligned}
\# \mathcal{N}_{1}^{c} & \leq(L+1) D_{f_{1}}(K, K+L)+(L+1)(\Delta+2(H+l) L \alpha \beta) \\
& \ll L \alpha^{1 / 3} \beta^{2 / 3}+\alpha^{-1 / 2}+L \Delta+2(H+l) L^{2} \alpha \beta \\
& \ll L K^{-\kappa / 3+o_{f}(1)}+K^{\kappa / 2+o_{f}(1)}+L p^{-\varepsilon / 4}+L^{2} p^{\varepsilon / 2} K^{-\kappa+o_{f}(1)} .
\end{aligned}
$$

Using (i) and (iii) we get $\# \mathcal{N}_{1}^{c} \ll L p^{-\varepsilon^{2}+o_{\varepsilon, f}(1)}$. Similarly, $\# \mathcal{N}_{2}^{c} \ll L p^{-\varepsilon^{2}+o_{\varepsilon, f}(1)}$. From (3.4) we get

$$
\# \mathcal{N}^{c} \ll_{\varepsilon, f} L p^{-\varepsilon^{2} / 2}
$$


For every integer $h \in \mathcal{H}$ we have

$$
\begin{aligned}
S_{f}\left(\chi_{1}, \chi_{2} ; K, L, l\right) & =\sum_{K<n \leq K+L} \chi_{1}(\lfloor f(n+h)\rfloor) \chi_{2}(\lfloor f(n+h+l)\rfloor)+O(h) \\
& =\sum_{n \in \mathcal{N}} \chi_{1}(\lfloor f(n+h)\rfloor) \chi_{2}(\lfloor f(n+h+l)\rfloor)+O_{\varepsilon, f}\left(p^{\varepsilon / 2}+\# \mathcal{N}^{c}\right) .
\end{aligned}
$$

Thus we have

$$
S_{f}\left(\chi_{1}, \chi_{2} ; K, L, l\right)=\frac{\Theta}{\# \mathcal{H}}+O_{\varepsilon, f}\left(p^{\varepsilon / 2}+L p^{-\varepsilon^{2} / 2}\right)
$$

where

$$
\Theta=\sum_{n \in \mathcal{N}} \sum_{h \in \mathcal{H}} \chi_{1}\left(\lfloor f(n)+\xi\rfloor+\left\lfloor h f^{\prime}(K)-\xi\right\rfloor\right) \chi_{2}\left(\left\lfloor f(n)+l f^{\prime}(K)+\xi\right\rfloor+\left\lfloor h f^{\prime}(K)-\xi\right\rfloor\right) .
$$

Now we divide $\mathcal{N}$ into two disjoint subsets:

$$
\mathcal{N}=\mathcal{M}_{1} \cup \mathcal{M}_{2} \quad \text { with } \mathcal{M}_{1} \cap \mathcal{M}_{2}=\varnothing,
$$

where

$$
\begin{aligned}
& \mathcal{M}_{1}=\left\{n \in \mathcal{N}:\{f(n)+\xi\}+\left\{l f^{\prime}(K)\right\} \geq 1\right\}, \\
& \mathcal{M}_{2}=\left\{n \in \mathcal{N}:\{f(n)+\xi\}+\left\{l f^{\prime}(K)\right\}<1\right\} .
\end{aligned}
$$

Then we have $\Theta=\Theta_{1}+\Theta_{2}$, where

$$
\begin{aligned}
\Theta_{1}=\sum_{n \in \mathcal{M}_{1}} \sum_{h \in \mathcal{H}} \chi_{1}\left(\lfloor f(n)+\xi\rfloor+\left\lfloor h f^{\prime}(K)-\xi\right\rfloor\right) \\
\times \chi_{2}\left(\lfloor f(n)+\xi\rfloor+\left\lfloor l f^{\prime}(K)\right\rfloor+1+\left\lfloor h f^{\prime}(K)-\xi\right\rfloor\right), \\
\Theta_{2}=\sum_{n \in \mathcal{M}_{2}} \sum_{h \in \mathcal{H}} \chi_{1}\left(\lfloor f(n)+\xi\rfloor+\left\lfloor h f^{\prime}(K)-\xi\right\rfloor\right) \\
\times \chi_{2}\left(\lfloor f(n)+\xi\rfloor+\left\lfloor l f^{\prime}(K)\right\rfloor+\left\lfloor h f^{\prime}(K)-\xi\right\rfloor\right) .
\end{aligned}
$$

Finally, we reduce the sums $\Theta_{1}$ and $\Theta_{2}$ into weighted double character sums and apply Lemma 2.4 to bound them. Let

$$
\begin{aligned}
\mathcal{U}_{i} & =\left\{u \in \mathbb{F}_{p}: u \equiv\lfloor f(n)+\xi\rfloor \quad(\bmod p) \text { for some } n \in \mathcal{M}_{i}\right\}, \quad i=1,2, \\
\mathcal{V} & =\left\{v \in \mathbb{F}_{p}: v \equiv\left\lfloor h f^{\prime}(K)-\xi\right\rfloor \quad(\bmod p) \text { for some } h \in \mathcal{H}\right\}
\end{aligned}
$$

and put

$$
\begin{array}{llll}
a_{u} & =\#\left\{n \in \mathcal{M}_{1}:\lfloor f(n)+\xi\rfloor \equiv u\right. & (\bmod p)\}, & u \in \mathcal{U}_{1}, \\
c_{u}=\#\left\{n \in \mathcal{M}_{2}:\lfloor f(n)+\xi\rfloor \equiv u\right. & (\bmod p)\}, & u \in \mathcal{U}_{2}, \\
b_{v}=\#\left\{h \in \mathcal{H}:\left\lfloor h f^{\prime}(K)-\xi\right\rfloor \equiv v\right. & (\bmod p)\}, & v \in \mathcal{V} .
\end{array}
$$


Thus we have

$$
\begin{aligned}
\Theta_{1} & =\sum_{u \in \mathcal{U}_{1}} \sum_{v \in \mathcal{V}} a_{u} b_{v} \chi_{1}(u+v) \chi_{2}\left(u+v+\left\lfloor l f^{\prime}(K)\right\rfloor+1\right), \\
\Theta_{2} & =\sum_{u \in \mathcal{U}_{2}} \sum_{v \in \mathcal{V}} c_{u} b_{v} \chi_{1}(u+v) \chi_{2}\left(u+v+\left\lfloor l f^{\prime}(K)\right\rfloor\right)
\end{aligned}
$$

In order to apply Lemma 2.4, we need to give the size of $\# \mathcal{U}_{1}, \# \mathcal{U}_{2}, \# \mathcal{V}, a_{u}, c_{u}$ and $b_{v}$. From the assumptions of Theorem 3.1 on $f^{\prime \prime}(x)$ we know that

$$
f^{\prime}(x)=x^{1-\kappa+o_{f}(1)} \quad \text { and } \quad f(x)=x^{2-\kappa+o_{f}(1)}, \quad x \rightarrow \infty .
$$

Since $\kappa<1$, the values of $\lfloor f(n)+\xi\rfloor(n \in \mathcal{N})$ are pairwise distinct, and in view of condition (ii) these values are of size at most $p^{1+o_{f}(1)}$. On the other hand, since $f^{\prime}(K)>1$, the values of $\left\lfloor h f^{\prime}(K)-\xi\right\rfloor(h \in \mathcal{H})$ are also pairwise distinct, and in view of (ii) these values are less than $p$. Therefore,

$$
a_{u} \leq p^{o_{f}(1)}, \quad c_{u} \leq p^{o_{f}(1)}, \quad b_{v}=1
$$

Then, from (3.3) we also have

$$
(\# \mathcal{V})=(\# \mathcal{H}) \geq p^{\varepsilon / 4}
$$

It remains to treat the cardinality of $\mathcal{U}_{1}$ and $\mathcal{U}_{2}$. From 3.5 we obtain

$$
\# \mathcal{N}=\left(\# \mathcal{M}_{1}\right)+\left(\# \mathcal{M}_{1}\right)=L+O_{\varepsilon, f}\left(L p^{-\varepsilon^{2} / 2}\right)
$$

This implies that

$$
\# \mathcal{M}_{1} \geq L p^{-\varepsilon^{2} / 16} \quad \text { or } \quad \# \mathcal{M}_{2} \geq L p^{-\varepsilon^{2} / 16} .
$$

Case 1. If $\# \mathcal{M}_{1} \geq L p^{-\varepsilon^{2} / 16}$ and $\# \mathcal{M}_{2}<L p^{-\varepsilon^{2} / 16}$. From (iii) we have

$$
\# \mathcal{U}_{1} \geq p^{1 / 2+\varepsilon-\varepsilon^{2} / 16+o_{f}(1)} \geq p^{1 / 2+\varepsilon / 4} .
$$

By Lemma 2.4 we have

$$
\Theta=\Theta_{1}+\Theta_{2} \ll_{\varepsilon}\left(\# \mathcal{U}_{1}\right)(\# \mathcal{V}) p^{-\varepsilon^{2} / 32}+\left(\# \mathcal{M}_{2}\right)(\# \mathcal{H}) \ll_{\varepsilon}(\# \mathcal{H}) L p^{-\varepsilon^{2} / 32}
$$

Case 2. If $\# \mathcal{M}_{1} \geq L p^{-\varepsilon^{2} / 16}$ and $\# \mathcal{M}_{2} \geq L p^{-\varepsilon^{2} / 16}$. From (iii) we also have

$$
\# \mathcal{U}_{1} \geq p^{1 / 2+\varepsilon / 4} \quad \text { and } \quad \# \mathcal{U}_{2} \geq p^{1 / 2+\varepsilon / 4}
$$

By Lemma 2.4 we have

$$
\Theta=\Theta_{1}+\Theta_{2} \ll_{\varepsilon}\left(\# \mathcal{U}_{1}\right)(\# \mathcal{V}) p^{-\varepsilon^{2} / 32}+\left(\# \mathcal{U}_{2}\right)(\# \mathcal{V}) p^{-\varepsilon^{2} / 32} \ll_{\varepsilon}(\# \mathcal{H}) L p^{-\varepsilon^{2} / 32}
$$

Now combining $3.6-3.8$ we immediately get Theorem 3.1 . 


\section{Proof of Theorem 1.2}

To get started, fix some constant $\eta>0$ to satisfy the inequality

$$
N^{\kappa-\eta} \geq p^{1 / 2+3 \eta}
$$

Since $N \geq p^{1 /(2 \kappa)+\varepsilon}$ and $\varepsilon<1 / 2$, then (4.1) is satisfied when choosing

$$
\eta=\frac{2 \varepsilon \kappa^{2}}{1+6 \kappa+2 \varepsilon \kappa}<\frac{\kappa}{2} .
$$

Moreover, (4.1) and $N \leq p^{1 /(2-\kappa)}$ imply that

$$
N^{\kappa / 2-\eta} \geq p^{3 \eta}
$$

We also let

$$
R=\left\lfloor N^{1+\eta-\kappa} \log ^{2} p\right\rfloor, \quad \Delta=p^{\eta / R}-1 .
$$

The first step is to prove that the bound

$$
S_{f}\left(\chi_{1}, \chi_{2} ; K, \Delta K, l\right)=\sum_{K<n \leq K+\Delta K} \chi_{1}(\lfloor f(n)\rfloor) \chi_{2}(\lfloor f(n+l)\rfloor) \ll_{\varepsilon, f} \Delta K p^{-\eta^{2} / 32}
$$

holds for all real $K$ with $N p^{-\eta} \leq K \leq N$. Noting that

$$
\Delta=\mathrm{e}^{\eta \log p / R}-1=(1+o(1)) \frac{\eta \log p}{R}=N^{\kappa-\eta-1}(\log p)^{-1+o(1)}, \quad p \rightarrow \infty .
$$

Then, for sufficiently large primes $p$ we have

$$
N^{\kappa-\eta-1} p^{-\eta} \leq \Delta \leq N^{\kappa-\eta-1}
$$

and therefore,

$$
\Delta K \leq N^{\kappa-\eta-1} K \leq K^{\kappa-\eta}
$$

From $K \geq N p^{-\eta}, 4.3$ and 4.5 we have

$$
\Delta K \geq K^{\kappa / 2} K^{1-\kappa / 2} N^{\kappa-\eta-1} p^{-\eta} \geq K^{\kappa / 2} N^{1-\kappa / 2} N^{\kappa-\eta-1} p^{-2 \eta} \geq K^{\kappa / 2} p^{\eta} .
$$

From $K \geq N p^{-\eta}$, 4.1) and (4.5) we also have

$$
\Delta K \geq K N^{\kappa-\eta-1} p^{-\eta} \geq N^{\kappa-\eta} p^{-2 \eta} \geq p^{1 / 2+\eta} .
$$

Combining (4.6)-(4.8) and the assumptions of Theorem 1.2 we conclude that

$$
K^{\kappa-\eta} \geq \Delta K \geq K^{\kappa / 2} p^{\eta}, \quad K \leq p^{1 /(2-\kappa)}, \quad \Delta K \geq p^{1 / 2+\eta} .
$$


Therefore, the assumptions of Theorem 3.1 are satisfied and we obtain (4.4).

Next, we decompose $S_{f}\left(\chi_{1}, \chi_{2} ; N\right)$ into $R+1$ sums. That is

$$
S_{f}\left(\chi_{1}, \chi_{2} ; N, l\right)=S_{f}\left(\chi_{1}, \chi_{2} ; N p^{-\eta}, l\right)+\sum_{j=0}^{R-1} S_{f}\left(\chi_{1}, \chi_{2} ; K_{j}, \Delta K_{j}, l\right),
$$

where

$$
K_{j}=N p^{-\eta+(\eta j) / R} .
$$

Since $K_{j}=K_{j-1}+\Delta K_{j-1}(j \geq 1)$. Thus, it follows from (4.4) and (4.9) that

$$
\begin{aligned}
S_{f}\left(\chi_{1}, \chi_{2} ; N, l\right) & =\sum_{j=0}^{R-1} S_{f}\left(\chi_{1}, \chi_{2} ; K_{j}, \Delta K_{j}, l\right)+O\left(N p^{-\eta}\right) \\
& \ll_{\varepsilon, f} \sum_{j=0}^{R-1} \Delta K_{j} p^{-\eta^{2} / 32}+N p^{-\eta} \ll_{\varepsilon, f} N p^{-\eta^{2} / 32} .
\end{aligned}
$$

This proves that the bound

$$
S_{f}\left(\chi_{1}, \chi_{2} ; N, l\right) \ll_{\varepsilon, f} N p^{-\delta}
$$

holds with some $\delta>0$. In particular, when $\eta$ takes the value as in 4.2 , one may take $\delta=2^{-9} \varepsilon^{2} \kappa^{4}$. This completes the proof of Theorem 1.2 .

5. Proofs of Theorems 1.3 and 1.4

We begin with Theorem 1.3 Let $(\dot{p})$ denote the Legendre symbol modulo $p$. Then we have

$$
\begin{aligned}
Q_{f}(N, l)= & \sum_{\substack{n \leq N \\
(\lfloor f(n)\rfloor\lfloor f(n+l)\rfloor, p)=1}} \frac{1}{4}\left(1+\left(\frac{\lfloor f(n)\rfloor}{p}\right)\right)\left(1+\left(\frac{\lfloor f(n+l)\rfloor}{p}\right)\right) \\
= & \sum_{n \leq N} \frac{1}{4}\left(1+\left(\frac{\lfloor f(n)\rfloor}{p}\right)\right)\left(1+\left(\frac{\lfloor f(n+l)\rfloor}{p}\right)\right)+O(W),
\end{aligned}
$$

where $W$ is defined by

$$
W=\#\{1 \leq n \leq N: p \mid\lfloor f(n)\rfloor\lfloor f(n+l)\rfloor\} .
$$

From the assumptions of Theorem 3.1 on $f^{\prime \prime}(x)$ we know that

$$
f(x)=x^{2-\kappa+o_{f}(1)}, \quad x \rightarrow \infty .
$$

Since $\kappa<1$ and $N<p^{1 /(2-\kappa)}$, the values of $\lfloor f(n)\rfloor(n=1,2, \ldots, N)$ are pairwise distinct, and these values are of size at most $p^{1+o_{f}(1)}$. Note that $0<l \leq p^{\varepsilon / 2}$, we also have the 
values of $\lfloor f(n+l)\rfloor(n=1,2, \ldots, N)$ are pairwise distinct and of size at most $p^{1+o_{f}(1)}$. Therefore,

$$
W \ll p^{o_{f}(1)} .
$$

It follows from $(5.1)$ and $(5.2)$ that

$$
\begin{aligned}
Q_{f}(N, l)= & \frac{N}{4}+\frac{1}{4} \sum_{n \leq N}\left(\frac{\lfloor f(n)\rfloor}{p}\right)+\frac{1}{4} \sum_{n \leq N}\left(\frac{\lfloor f(n+l)\rfloor}{p}\right) \\
& +\frac{1}{4} \sum_{n \leq N}\left(\frac{\lfloor f(n)\rfloor}{p}\right)\left(\frac{\lfloor f(n+l)\rfloor}{p}\right)+O\left(p^{o_{f}(1)}\right) .
\end{aligned}
$$

From Proposition 1.1 and Theorem 1.2 we immediately get

$$
Q_{f}(N, l)=\frac{N}{4}+O_{\varepsilon, f}\left(N p^{-\theta}\right)
$$

where $\theta$ is defined as in $(1.3)$. This proves Theorem 1.3 .

Now we prove Theorem 1.4 . Noting that

$$
\frac{\phi(p-1)}{p-1} \sum_{\nu \mid p-1} \frac{\mu(\nu)}{\phi(\nu)} \sum_{\text {ord } \chi=\nu} \chi(n)= \begin{cases}1 & \text { if } n \text { is a primitive root modulo } p \\ 0 & \text { otherwise }\end{cases}
$$

where $\mu$ is the Möbious function, and the summation $\sum_{\text {ord } \chi=\nu}$ is taken over all the characters $\chi$ modulo $p$ of order $\nu$. Thus we have

$$
\begin{aligned}
G_{f}(N, l)= & \left(\frac{\phi(p-1)}{p-1}\right)^{2} \sum_{\nu_{1} \mid p-1} \sum_{\nu_{2} \mid p-1} \frac{\mu\left(\nu_{1}\right) \mu\left(\nu_{2}\right)}{\phi\left(\nu_{1}\right) \phi\left(\nu_{2}\right)} \sum_{\text {ord } \chi_{1}=\nu_{1}} \sum_{\text {ord } \chi_{2}=\nu_{2}} \\
& \times \sum_{n \leq N} \chi_{1}(\lfloor f(n)\rfloor) \chi_{2}(\lfloor f(n+l)\rfloor) \\
= & \left(\frac{\phi(p-1)}{p-1}\right)^{2} N+\left(\frac{\phi(p-1)}{p-1}\right)^{2} \sum_{\substack{\nu_{1} \mid p-1 \\
\nu_{1}>1}} \frac{\mu\left(\nu_{1}\right)}{\phi\left(\nu_{1}\right)} \sum_{\text {ord } \chi_{1}=\nu_{1}} \sum_{\substack{n \leq N \\
(\lfloor f(n+l)\rfloor, p)=1}} \chi_{1}(\lfloor f(n)\rfloor) \\
& +\left(\frac{\phi(p-1)}{p-1}\right)^{2} \sum_{\substack{\nu_{2} \mid p-1 \\
\nu_{2}>1}} \frac{\mu\left(\nu_{2}\right)}{\phi\left(\nu_{2}\right)} \sum_{\text {ord } \chi_{2}=\nu_{2}} \sum_{\substack{n \leq N \\
(\lfloor f(n)\rfloor, p)=1}} \chi_{2}(\lfloor f(n+l)\rfloor) \\
+ & \left(\frac{\phi(p-1)}{p-1}\right)^{2} \sum_{\substack{\nu_{1} \mid p-1 \\
\nu_{1}>1}} \sum_{\nu_{2} \mid p-1} \frac{\mu\left(\nu_{1}\right) \mu\left(\nu_{2}\right)}{\phi\left(\nu_{1}\right) \phi\left(\nu_{2}\right)} \sum_{\text {ord } \chi_{1}=\nu_{1} \text { ord } \chi_{2}=\nu_{2}} \\
& \times \sum_{n \leq N} \chi_{1}(\lfloor f(n)\rfloor) \chi_{2}(\lfloor f(n+l)\rfloor)+O(W) .
\end{aligned}
$$


From Proposition 1.1 and 5.2 we have

$$
\sum_{\substack{n \leq N \\(\lfloor f(n+l)\rfloor, p)=1}} \chi_{1}(\lfloor f(n)\rfloor)=\sum_{n \leq N} \chi_{1}(\lfloor f(n)\rfloor)+O(W)=O_{\varepsilon, f}\left(N p^{-\theta}\right),
$$

and

$$
\sum_{\substack{n \leq N \\(\lfloor f(n)\rfloor, p)=1}} \chi_{2}(\lfloor f(n+l)\rfloor)=\sum_{n \leq N} \chi_{2}(\lfloor f(n+l)\rfloor)+O(W)=O_{\varepsilon, f}\left(N p^{-\theta}\right) .
$$

From Theorem 1.2 we also have

$$
\sum_{n \leq N} \chi_{1}(\lfloor f(n)\rfloor) \chi_{2}(\lfloor f(n+1)\rfloor)=O_{\varepsilon, f}\left(N p^{-\theta}\right) .
$$

Combining (5.3)-(5.6) we immediately get

$$
G_{f}(N, l)=\left(\frac{\phi(p-1)}{p-1}\right)^{2} N+O_{\varepsilon, f}\left(\left(\frac{\phi(p-1)}{p-1}\right)^{2} 4^{\omega(p-1)} N p^{-\theta}\right) .
$$

This proves Theorem 1.4 .

\section{Remarks}

In this paper we obtain a non-trivial bound for $S_{f}\left(\chi_{1}, \chi_{2} ; N, l\right)$ by using the method of [5, 14] (also see 3, 4]). In detail, we decompose the sum $S_{f}\left(\chi_{1}, \chi_{2} ; N, l\right)$ into character sums over short intervals and finally reduce them into certain double character sums. In these processes, the difference lies in Lemma 2.3 and Theorem 3.1 .

We also remark that Theorem 1.2 holds when $l$ is small. In the proof of Theorem 3.1 , we must ensure that the upper bound of $(3.2)$ is less than 1 and $\# \mathcal{N}^{c}=o(L)$ in 3.5 . Therefore, if $l$ is large, we may not be able to find suitable $K$ and $L$ to apply to the proof of Theorem 1.2. Thus, we restrict that $0<l \leq p^{\varepsilon / 2}$ throughout this paper.

\section{Acknowledgments}

The authors express their gratitude to the referee for his/her helpful and detailed comments.

\section{References}

[1] R. C. Baker and W. D. Banks, Character sums with Piatetski-Shapiro sequences, Q. J. Math. 66 (2015), no. 2, 393-416. 
[2] W. D. Banks, M. Z. Garaev, D. R. Heath-Brown and I. E. Shparlinski, Density of non-residues in Burgess-type intervals and applications, Bull. Lond. Math. Soc. 40 (2008), no. 1, 88-96.

[3] W. D. Banks and I. E. Shparlinski, Non-residues and primitive roots in Beatty sequences, Bull. Austral. Math. Soc. 73 (2006), no. 3, 433-443.

[4] - Short character sums with Beatty sequences, Math. Res. Lett. 13 (2006), no. $4,539-547$.

[5] _ Multiplicative character sums with twice-differentiable functions, Q. J. Math. 60 (2009), no. 4, 401-411.

[6] A. A. Bennett, Consecutive quadratic residues, Bull. Amer. Math. Soc. 32 (1926), no. $3,283-284$.

[7] S. D. Cohen, Consecutive primitive roots in a finite field, Proc. Amer. Math. Soc. 93 (1985), no. 2, 189-197.

[8] _ Consecutive primitive roots in a finite field II, Proc. Amer. Math. Soc. 94 (1985), no. 4, 605-611.

[9] M. Z. Garaev, A note on the least quadratic non-residue of the integer-sequences, Bull. Austral. Math. Soc. 68 (2003), no. 1, 1-11.

[10] A. Hildebrand, On consecutive kth power residues, Monatsh. Math. 102 (1986), no. 2, $103-114$.

[11] P. Hummel, On consecutive quadratic non-residues: A conjecture of Issai Schur, J. Number Theory 103 (2003), no. 2, 257-266.

[12] Y.-K. Lau and J. Wu, On the least quadratic non-residue, Int. J. Number Theory 4 (2008), no. 3, 423-435.

[13] W. M. Schmidt, Equations over Finite Fields: An elementary approach, Lecture Notes in Mathematics 536, Springer-Verlag, New York, 1976.

[14] I. E. Shparlinski and M. Technau, Kloosterman sums with twice-differentiable functions, Funct. Approx. Comment. Math. 63 (2020), no. 1, 113-124.

[15] J. Tanti and R. Thangadurai, Distribution of residues and primitive roots, Proc. Indian Acad. Sci. Math. Sci. 123 (2013), no. 2, 203-211.

[16] E. Vegh, Pairs of consecutive primitive roots modulo a prime, Proc. Amer. Math. Soc. 19 (1968), 1169-1170. 
Mengyao Jing and Huaning Liu

Research Center for Number Theory and its Applications, School of Mathematics, Northwest University, Xi'an 710127, Shaanxi, China

E-mail addresses: myjing@stumail.nwu.edu.cn, hnliu@nwu.edu.cn 